\title{
Invisible but Unpleasant: Unconscious emotional processing of trypophobic objects
}

\author{
Kyoshiro Sasaki $^{1,2,3^{*}}$, Katsumi Watanabe ${ }^{1}$, and Yuki Yamada ${ }^{2}$ \\ ${ }^{1}$ Waseda University, Tokyo, Japan \\ ${ }^{2}$ Kyushu University, Fukuoka, Japan \\ ${ }^{3}$ Japan Society for the Promotion of Science, Tokyo, Japan
}

Running head: Trypophobic discomfort without awareness

*Corresponding author

Kyoshiro Sasaki

Address: Faculty of Science and Engineering, Waseda University,

3-4-1, Ohkubo, Shinjuku, Tokyo, 169-8555, Japan.

E-mail: kyoshiro0920@gmail.com

TEL \& FAX: +81-3-5286-3335 


\begin{abstract}
Clusters of round objects (e.g., lotus seedpods) induce strong discomfort (trypophobic discomfort). A previous study has shown that trypophobic discomfort stems not only from middle but also low spatial frequency information, which should be rapidly transmitted to the amygdala via the superior colliculus and pulvinar pathway. Based on this finding, we hypothesized that trypophobic discomfort would be evoked by invisible trypophobic images; the present study examined this using a backward masking technique. We found that trypophobic images were reported as unpleasant significantly above the chance level irrespectively of visibility of the image (Experiments 1 and 2). On the other hand, detection sensitivity for trypophobic images was lower than that for neutral images (Experiment 3). Taken together, our findings suggest that emotional information about trypophobic objects is processed unconsciously although this information does not promote detection.
\end{abstract}

Keywords: Trypophobia; Emotion; Awareness; Consciousness; Backward masking 


\section{Introduction}

Clusters of round objects (e.g., lotus seed pods) induce strong discomfort. This discomfort is referred to as trypophobia. The word trypophobia consists of the Greek word "trypo" (i.e., hole) and the English word phobia (Abbasi, 2011). Trypophobia is a hot topic on the Internet that has received widespread attention. Actually, a trypophobia Facebook page that has existed since 2009 had 13,640 followers in 2018, and many pictures have been uploaded on the page. While trypophobia draws great interest, this phobia represents a significant problem for some people (e.g., Martínez-Aguayo et al., 2018). Thus, it is important to reveal the mechanism of trypophobia and develop methods to control trypophobia based on this mechanism.

Scientific research on trypophobia began in 2013 with Cole and Wilkins. They examined visual characteristics of trypophobic images and revealed that contrast energy at the midrange spatial frequency is higher in trypophobic images than in neutral images. They also found that this spectrum was similar to that of poisonous animals (e.g., the blue-ringed octopus). Considering that high-contrast energy at the midrange spatial frequency involves visual discomfort (Fernandez \& Wilkins, 2008), these findings seemed to suggest that midrange spatial frequency components play key roles in trypophobia.

However, subsequent studies have demonstrated that trypophobia is not elicited only by midrange spatial frequency components. Most natural images have a $1 / f$ amplitude spectrum and images deviating from this spectrum induce visual discomfort (Fernandez \& Wilkins, 2008; O’Hare \& Hibbard, 2011). As we mentioned above, trypophobic images have excess energy at the midrange spatial frequency and therefore deviate from the $1 / f$ spectrum (Cole \& Wilkins, 2013). Therefore, 
trypophobic discomfort should be reduced if the excess energy were removed from the trypophobic image; however, this is not actually true. Le and colleagues filtered trypophobic images such that the images had a $1 / f$ spectrum and revealed that the filtered images still induced discomfort (Le, Cole, \& Wilkins, 2015). Moreover, even when midrange spatial frequency components were removed, trypophobic discomfort did not decrease (Sasaki et al., 2017). Sasaki et al. have also revealed that strong discomfort was induced not only by original and mid-pass filtered trypophobic images but also by low-pass filtered trypophobic images. These findings suggest that low spatial frequency as well as middle spatial frequency components induce trypophobic discomfort. This further raises a possibility: We could process trypophobic objects rapidly and unconsciously.

Is emotion processed out of our awareness? This issue has been addressed by use of a famous emotional phenomenon: a visible prime facilitates response to a subsequent target when emotional valences are congruent between the prime and the target (affective priming: e.g., Fazio et al., 1986; Hermans, De Houwer, \& Eelen, 2001; Murphy \& Zajonc, 1993). This affective priming occurs even when the prime is subliminally presented (e.g., Ghuman \& Bar, 2006; Murphy \& Zajonc, 1993; Rotteveel et al., 2001; Sweeny et al., 2009), indicating that unconscious information of the emotional prime influences subsequent processing. In addition, unconscious emotional processing of negative stimuli often prevails against that of positive ones. For example, snake- and spider-phobic people feel negative emotions from masked images of snakes and spiders, respectively (Öhman \& Soares, 1994). Moreover, subliminal negative words are more correctly categorized than subliminal positive ones (e.g., Dijksterhuis \& Aarts, 2003). Taken together, emotional information, particularly if negative, is 
processed unconsciously.

Previous studies have examined neural processing of low spatial frequency threat objects (e.g., Vuilleumier et al., 2003; Winston, Vuilleumier, \& Dolan, 2003). In these studies, it was proposed that threat-related cues of low spatial frequency are processed in the superior colliculus and pulvinar, and this information is rapidly transmitted to the amygdala. This colliculus-pulvinar pathway contributes to rapid and unconscious processing of threat objects (e.g., Bar, Neta, \& Linz, 2006; Kawai \& He, 2016; Tsuchiya, Moradi, \& Felsen, 2009). Low spatial frequency components induce trypophobic discomfort (Sasaki et al., 2017), and thus these cues of trypophobic discomfort should be rapidly transmitted to the amygdala via the colliculus-pulvinar pathway. Therefore, trypophobic objects are possibly judged as unpleasant without awareness.

The present study aimed to examine whether trypophobic discomfort would occur unconsciously using the backward masking technique. If trypophobic discomfort occurred unconsciously, trypophobic images would be judged as causing discomfort, without visual awareness. If trypophobic discomfort only occurs consciously, then judgment of invisible trypophobic images would not be accurate.

\section{Experiment 1}

\section{Methods}

Participants. Thirteen volunteers participated in the experiment. All participants were unaware of the aim of this experiment and reported that they had normal visual functions. The study protocol was approved by the ethics committees of Waseda University. The experiment was conducted according to the guidelines stipulated in the 
Declaration of Helsinki. We told the participants the following: We would use emotional stimuli, and thus they could stop the experiment whenever they became distressed. We obtained written informed consent from all participants prior to the experiments.

Apparatus. The stimuli were presented on a 22-inch monitor. The resolution was 1920 $\times 1080$ pixels, and the refresh rate was $100 \mathrm{~Hz}$. The presentation of the stimuli and data were controlled by a computer. The stimuli were generated by MATLAB (The MathWorks, Inc., Natick, MA, USA) with the Psychtoolbox extension (Brainard, 1997; Pelli, 1997).

Stimuli and Procedure. The viewing distance was $57 \mathrm{~cm}$. Stimuli consisted of a fixation mark, target images, and scrambled images. The fixation mark was a white circle (radius was $0.5 \mathrm{deg}$ ). The target images were 20 trypophobic and 20 neutral images $(20 \times 20 \mathrm{deg})$. Fifteen trypophobic images were selected from the ones used in Cole and Wilkins (2013) and Le et al. (2015). The rest were obtained from a Google search for "trypophobia" in English and Japanese. A preliminary survey revealed that the trypophobic trait (Chaya et al., 2016; Imaizumi et al., 2015; Le et al., 2016) significantly predicted discomfort from these trypophobic images $(\beta=-.55, p<.001)$. Thus, the new stimulus set was confirmed to induce trypophobia. Twenty neutral images were selected from the ones used in Cole and Wilkins (2013) and Le et al. (2015). The scrambled images were made by dividing each of the trypophobic and neutral images into a $128 \times 128$ grid and randomly reordering the segments.

The experiment was conducted in a darkened room. The participants sat in front of the display and their head position was stabilized with a head and chin rest. Figure 1 shows the timeline of a trial. The participants initiated each trial by pressing 
the spacebar. Then, the fixation mark was presented for $1500 \mathrm{~ms}$. After the fixation mark disappeared, the target image (trypophobic or neutral) was presented for 10, 40, or $80 \mathrm{~ms}$. Then, the scrambled image, whose original image corresponded with the target image, was presented for $250 \mathrm{~ms}$. After the scrambled image disappeared, participants were asked to judge whether the target image was unpleasant or neutral as soon as possible. After the judgment, participants rated the visibility of the target images with a 4-point scale: 1 (I did not see the image at all), 2 (I saw a glimpse of something, but do not know what it was), 3 (I saw something, and think I can determine what it was), and 4 (I saw the image clearly). Each participant performed 120 trials: two target types (trypophobic and neutral) $\times$ three target durations $(10,40$, and $80 \mathrm{~ms}) \times 20$ images.

Data analysis. When the visibility score was 1 or 2 , we assigned this trial into the invisible condition. On the other hand, the rest was assigned into the visible condition. We calculated the proportion of correct responses (i.e., the trypophobic image was judged as unpleasant or the neutral image was judged as neutral) for each condition. We excluded persons whose correct responses to the trypophobic images were below 0.5 in the visible condition because they did not feel trypophobic discomfort. To examine whether the proportion of correct responses was significantly different from the chance level (0.5), we conducted one-sample $t$-tests and calculated Cohen's $d z \mathrm{~s}$. Moreover, we conducted a two-way analysis of variance (ANOVA) on the correct response with the target type (trypophobic or neutral) and the target visibility (visible or invisible) to examine whether the correct response was different between the target types and calculated $\eta_{p}{ }^{2} \mathrm{~s}$. 


\section{Results and discussion}

One female participant was excluded according to the criteria indicated in Data analysis, and thus we analyzed the data of the 12 remaining participants ( 9 males and 3 females, mean age \pm S.E.M. $=21.25 \pm 0.84)$. Figure 2 shows the results of Experiment 1. In the visible condition, the proportion of correct responses for both target types was significantly higher than the chance level (trypophobic: $t(11)=11.23$, $p<.001$, Cohen's $d z=3.24$; neutral: $t(11)=51.92, p<.001$, Cohen's $d z=14.99)$. On the other hand, in the invisible condition, the proportion of correct responses was above the chance level only in the trypophobic condition $(t(11)=6.33, p<.001$, Cohen's $d z=1.83$, while it was not significantly different from the chance level in the neutral condition $(t(11)=1.45, p=.18$, Cohen's $d z=0.42)$. Moreover, ANOVA revealed a significant main effect of target visibility $\left(F(1,11)=49.01, p<.001, \eta_{p}{ }^{2}\right.$ $=.82)$, while the main effect of target type was not significant $(F(1,11)=0.58, p=.46$, $\left.\eta_{p}{ }^{2}=.05\right)$. Additionally, the interaction was significant $\left(F(1,11)=14.80, p=.003, \eta_{p}{ }^{2}\right.$ $=.57)$. Post-hoc tests revealed that the simple main effect of target type was significant in the invisible condition $\left(F(1,22)=8.99, p=.007, \eta_{p}{ }^{2}=.29\right)$, while this effect was not significant in the visible condition $\left(F(1,22)=3.29, p=.08, \eta_{p}{ }^{2}=.13\right)$. Moreover, the simple main effect of target visibility was not significant in the trypophobic condition $\left(F(1,22)=0.27, p=.61, \eta_{p}^{2}=.01\right)$, whereas this effect was significant in the neutral condition $\left(F(1,22)=49.12, p<.001, \eta_{p}{ }^{2}=.69\right)$. These results suggest that trypophobic images were judged as unpleasant without awareness.

One might argue that the scrambled images induced trypophobic discomfort and this became a cue for judgment of the target image. To address this issue, we asked another 19 participants to rate the emotional valence of the scrambled images with a 
9-point scale (1: highly unpleasant, 9: highly pleasant). As a result, no significant difference in emotional valence was found between the trypophobic and neutral scrambled images $(t(18)=0.33, p=.75$, Cohen's $d z=0.08)$. Thus, the scrambled images were unlikely to be the cues used for judgment.

A previous study revealed that color information influences processing of emotional images (Nomura \& Yotsumoto, 2018). Perhaps, the effect of color mediated the results of Experiment 1. Thus, Experiment 2 used achromatic images and examined whether the results of Experiment 1 could be replicated in this case.

\section{Experiment 2}

\section{Methods}

Participants, Apparatus, Stimuli, Procedure, and Data analysis. We recruited 16 volunteers. This was because four persons did not feel trypophobic discomfort even when the images were visible (the rate of their correct responses for the trypophobic images was below 0.5 in the visible condition). Thus, we excluded them and added another four volunteers to match the number of participants between Experiments 1 and 2. Finally, we analyzed the data from 12 volunteers ( 5 males and 7 females, mean age \pm S.E.M. $=20.08 \pm 0.60)$. The methods were identical to Experiment 1 except that the images were grayscaled (mean gray level $=114)$.

\section{Results and discussion}

Figure 3 shows the results of Experiment 2. In the visible condition, the proportion of correct responses in both target types was significantly higher than the chance level (trypophobic: $t(11)=1.22, p=.001$, Cohen's $d z=1.73$; neutral: $t(11)=$ 
10.00, $p<.001$, Cohen's $d z=2.89$ ). Moreover, in the invisible condition, the proportion of correct responses was above the chance level in both types of images $(t(11)=3.55, p=.004$, Cohen's $d z=1.03 ; t(11)=2.32, p=.04$, Cohen's $d z=0.67)$. Moreover, ANOVA revealed a significant main effect of visibility $(F(1,11)=19.07, p$ $\left.=.001, \eta_{p}{ }^{2}=.63\right)$. On the other hand, the main effect of target type and the interaction were not significant (target types: $F(1,11)=0.001, p=.97, \eta_{p}{ }^{2}=.00$; interaction: $F(1$, $\left.11)=1.27, p=.28, \eta_{p}{ }^{2}=.10\right)$. We replicated the results of Experiment 1: Trypophobic images were judged as unpleasant without awareness even when the images were monochrome. These results suggest that color did not mediate the effects in Experiment 1. In contrast to the results of Experiment 1, the correct responses to neutral images were significantly higher than the chance level. However, the effect sizes were not high, and thus this result should be negligible here.

This unconscious information might also contribute to detection of trypophobic objects. Actually, previous studies have revealed that unconscious emotional information often promotes detection of objects (e.g., Calvo, Esteves, \& Esteves, 2005; Dijksterhuis \& Aarts, 2003; Stein et al., 2014; Tsuchiya et al., 2009; Yang, Zald, \& Blake, 2007). Moreover, trypophobic images capture visual attention (Shirai, Banno, \& Ogawa, in press) and bottom-up attention is related to conscious perception (for a review, see Dehaene et al., 2006). We examined these issues in Experiment 3 by measuring detection sensitivity to trypophobic and neutral images.

\section{Experiment 3}

\section{Methods}

Participants, Apparatus, Stimuli, Procedure, and Data analysis. Twenty volunteers 
participated in the experiment (13 males and 7 females, mean age \pm S.E.M. $=19.8 \pm$ 0.33). The apparatus, stimuli, and procedure were identical to Experiment 1 except in the following respects. Figure 4 shows the timeline of a trial in Experiment 3. We presented not only trypophobic and neutral images as target stimuli but also white noise $(20 \times 20 \mathrm{deg})$ as a filler stimulus. The target (filler) duration was 10 or $80 \mathrm{~ms}$. We used Mondrian images consisting of a clutter of approximately 200 colorful rectangles as masks. The center of each rectangle was within an imaginary square, whose sides were $20 \mathrm{deg}$. The task was to identify whether the target stimulus was a picture or white noise instead of making an emotional judgment. Each participant performed 160 trials: 40 target images (20 trypophobic and 20 neutral images), 40 fillers, and two target durations (10 or $80 \mathrm{~ms})$.

To examine the detection sensitivity of each target type (trypophobic or neutral), we calculated the sensitivity ( $\left.d^{\prime}\right)$ and bias indices $(c)$ based on signal detection theory. We conducted one-sample $t$-tests to confirm whether $d$ ' and $c$ were significantly different from zero. Moreover, we performed a two-way ANOVA on $d$ ' and $c$ with the target type (trypophobic or neutral) and the target duration (10 or $80 \mathrm{~ms}$ ) as within participant factors.

\section{Results and discussion}

Figure 5 shows the results of Experiment 3. The $d^{\prime}$ of the trypophobic images was not significantly different from zero in the 10 -ms condition $(t(19)=1.26, p=.22$, Cohen's $d z=0.28)$. The other $d$ 's were significantly higher than zero (10-ms neutral: $t(19)=3.79, p=.001$, Cohen's $d z=0.85 ; 80$-ms trypophobic: $t(19)=28.43, p<.001$, Cohen's $d z=6.36 ; 80$-ms neutral: $t(19)=44.35, p<.001$, Cohen's $d z=9.92$ ). The 
ANOVA of the $d$ ' revealed that the main effect of target type was significant $(F(1,19)$ $\left.=7.72, p=.01, \eta_{p}^{2}=.28\right)$, indicating that $d^{\prime}$ 'was higher in the neutral condition than in the trypophobic condition. The main effect of target duration was also significant $(F(1$, $\left.19)=609.69, p<.001, \eta_{p}{ }^{2}=.97\right)$. The interaction was not significant $(F(1,19)=2.09$, $\left.p=.17, \eta_{p}^{2}=.10\right)$

In the 10 -ms condition, the $c$ s were significantly lower than zero in both image types (trypophobic: $t(19)=2.10, p=.05$, Cohen's $d z=0.47$; neutral: $t(19)=$ 2.92, $p=.009$, Cohen's $d z=0.65$ ), indicating that the participants' judgment was biased toward white noise in the 10-ms condition. On the other hand, the $c \mathrm{~s}$ for the trypophobic image were significantly above zero in both image types $(t(19)=2.27, p$ $=.04$, Cohen's $d z=0.51)$. The $c$ s for the neutral image were not significantly different from zero $(t(19)=1.95, p=.07$, Cohen's $d z=0.44)$. The ANOVA of the $c$ revealed that the main effect of target type was significant $\left(F(1,19)=7.72, p=.01, \eta_{p}{ }^{2}=.28\right)$, indicating that $d$ ' was higher in the neutral condition than in the trypophobic condition. That is, the participants' judgment was biased toward the target in the trypophobic condition compared to the neutral one. The main effect of the target duration was also significant $\left(F(1,19)=9.73, p<.001, \eta_{p}{ }^{2}=.34\right)$. The interaction was not significant $\left(F(1,19)=2.09, p=.17, \eta_{p}^{2}=.10\right)$.

Briefly, detection sensitivity to the trypophobic image was lower than that to the neutral image. Thus, these findings suggest that unconscious emotional information does not promote detection of trypophobic objects. Additionally, the results rule out the possibility that the reason why trypophobic images were judged as unpleasant without awareness in the results of Experiments 1 and 2 was due to ease of detection. These results contradicted our prediction. We discuss these issues in detail in the 
General Discussion.

\section{General discussion}

The present study examined unconscious emotional processing of trypophobic images by using a backward masking technique. Experiment 1 demonstrated that invisible trypophobic images were judged as unpleasant. Similar results were obtained even when the images were achromatic (Experiment 2). This indicated that this phenomenon did not depend on chromatic information. Experiment 3 showed that detection sensitivity in the trypophobic images was low. Taken together, our findings suggest that emotional information of the trypophobic object is processed unconsciously but this information does not promote detection. That is, trypophobic objects are unpleasant even when they are invisible.

Why were trypophobic objects judged as unpleasant without visual awareness? Several emotional models assume two pathways: Low and high roads (LeDoux, 1998; Tamietto \& de Gelder, 2010; Vuilleumier, 2005). The high road undertakes processing of detailed visual information involving conscious experience. On the other hand, the low road relates to coarse but fast visual processing, and this processing contributes to unconscious emotional processing. In these models, low spatial frequency information plays a key role. Specifically, low spatial frequency information of emotional visual events is rapidly conveyed to the amygdala via the colliculus-pulvinar pathway (e.g., Vuilleumier et al., 2003; Winston, Vuilleumier, \& Dolan, 2003). As a result, emotional reactions occur before consciousness of the events. Low spatial frequency information of the trypophobic objects may have been transmitted to the amygdala, and thus unpleasantness was evoked prior to conscious 
experience of the objects. Actually, low-pass filtered images of trypophobic objects are sufficient to induce trypophobic discomfort (Sasaki et al., 2017) and this previous finding supports our notion about unconscious emotional processing of trypophobic objects.

We found that detection sensitivity to trypophobic objects is low in comparison with neutral images. Previous studies have shown that detection sensitivity to emotional stimuli is higher than that to neutral stimuli (e.g., Calvo et al., 2005; Dijksterhuis \& Aarts, 2003; Stein et al., 2014; Tsuchiya et al., 2009; Yang et al., 2007). Thus, the present findings seem to be contrary to previous ones. Although the reason for this contradiction is unclear at this time, detailed visual information (e.g., high spatial frequency information) of trypophobic objects might be involved. As we mentioned above, the high road is assumed to process detailed visual information generating conscious experience (LeDoux, 1998; Tamietto \& de Gelder, 2010; Vuilleumier, 2005). Perhaps, detailed visual information of trypophobic images is not processed smoothly compared with that of neutral images. As a result, trypophobic images might have been difficult to detect in the present study. To resolve this issue, further direct investigations would be necessary.

Previous research has revealed that trypophobic images have excess energy at the midrange spatial frequency compared with neutral images (Cole \& Wilkins, 2013). Although subsequent studies have shown that trypophobia does not only involve the middle spatial frequency (Le et al., 2015; Sasaki et al., 2017), excess energy at the midrange spatial frequency possibly contributes to unconscious emotional processing of trypophobic images. To confirm this possibility, we conducted a similar spectrum analysis to that of Cole and Wilkins (2013). The results showed no difference in 
energy between trypophobic and neutral images $(p s>.11)$. Thus, at least, excess energy at the midrange spatial frequency did not involve trypophobic discomfort induced by invisible images.

One might argue that participants' judgment was biased toward unpleasant responses and this response bias mediated the results of Experiments 1 and 2. In this case, correct responses to trypophobic images would be above the chance level irrespective of unconscious emotional processing. However, if judgments were biased toward unpleasant responses, correct responses to neutral images would be below the chance level; however, this was not true in the present study. Therefore, we rejected the possibility that response bias mediated the results of Experiments 1 and 2 .

The present study sheds light on negative reactions to invisible images of trypophobic objects. Previous studies revealed and discussed rapid and unconscious processing of harmful objects (e.g., Bar, Neta, \& Linz, 2006; Kawai \& He, 2016; Tsuchiya, Moradi, \& Felsen, 2009). This system is ecologically adaptive because we can avoid such objects without directly interacting with them, which might increase survival rates. From this perspective, negative reactions to invisible images of trypophobic objects seems to be strange since most of them are not harmful. A previous study has demonstrated that the spectrum of the trypophobic images is similar to that of poisonous animals and speculated that this visual property induces an avoidance reaction (Cole \& Wilkins, 2013). However, a subsequent study showed that trypophobic objects were not associated with poisonous animals (Can, Zhuoran, \& Zheng, 2017). On the other hand, Yamada and Sasaki (2017) recently proposed a new possible explanation: The "involuntary protection against dermatosis" (IPAD) hypothesis. The IPAD hypothesis proposes that trypophobic objects elicit negative 
emotion because their appearance is associated with skin diseases, and hence an avoidance reaction to pathogens is evoked. The IPAD hypothesis is supported by empirical evidence demonstrating that trypophobia involves disgust, particularly core disgust (i.e., emotional avoidance of pathogen infection) (Imaizumi et al., 2016; Kupfer \& Le, 2018; Vlok-Barnard \& Stein, 2017). Based on this, negative reactions to invisible trypophobic objects might stem from involuntary protection against dermatosis.

Previous studies assumed a psychological system of pathogen avoidance including a sensory and perceptual process (behavioral immune system: Park, Schaller, \& Crandall, 2007; Schaller, 2011; Schaller \& Park, 2011). In this system, perceptual cues of infectious pathogens evoke aversive emotion, and then this emotion facilitates avoidance reaction. Based on the IPAD hypothesis (Yamada \& Sasaki, 2017), trypophobia can be considered as an overreaction of the behavioral immune system because most of trypophobic objects are harmless and thus not infectious pathogens. In line with this notion, our findings possibly indicate that malfunction of the behavioral immune system occurs at the unconscious stage of mental processing. Further experimental examinations of trypophobia from the perspective of pathogen avoidance might also be beneficial for revealing how the behavioral immune system would commit errors and comprehensively understanding the cognitive mechanism underlying the behavioral immune system. 


\section{References}

Abbasi, J. (2011). Is trypophobia a real phobia? Popular Science. Retrieved from http: //www.popsci.com/trypophobia

Bar, M., Neta, M., \& Linz, H. (2006). Very first impressions. Emotion, 6, 269-278.

Calvo, M., Esteves, F., \& Esteves, F. (2005). Detection of emotional faces: low perceptual threshold and wide attentional span. Visual Cognition, 12, 13-27.

Can, W., Zhuoran, Z., \& Zheng, J. (2017). Is trypophobia a phobia? Psychological Reports, 120, 206-218.

Chaya, K., Xue, Y., Uto, Y., Yao, Q., \& Yamada, Y. (2016). Fear of eyes: triadic relation among social anxiety, trypophobia, and discomfort for eye cluster. PeerJ, 4:e1942.

Cole, G. G., \& Wilkins, A. J. (2013). Fear of holes. Psychological Science, 24, $1980-1985$.

Dehaene, S., Changeux, J.-P., Naccache, L., Sackur, J., \& Sergent, C. (2006). Conscious, preconscious, and subliminal processing: a testable taxonomy. Trends in Cognitive Sciences, 10, 204-211.

Dijksterhuis, A., \& Aarts, H. (2003). On wildebeests and humans: The Preferential Detection of Negative Stimuli. Psychological Science, 14, 14-18.

Fazio, R. H., Sanbonmatsu, D. M., Powell, M. C., \& Kardes, F. R. (1986). On the Automatic Activation of Attitudes. Journal of Personality and Social Psychology, 50, 229-238.

Fernandez, D., \& Wilkins, A. J. (2008). Uncomfortable images in art and nature. Perception, 37, 1098-1113.

Ghuman, A. S., \& Bar, M. (2006). The Influence of Nonremembered Affective 
Associations on Preference. Emotion, 6, 215-223.

Hermans, D., De Houwer, J., \& Eelen, P. (2001). A time course analysis of the affective priming effect. Cognition and Emotion, 15, 143-165.

Imaizumi, S., Furuno, M., Hibino, H., \& Koyama, S. (2016). Trypophobia is predicted by disgust sensitivity, empathic traits, and visual discomfort. SpringerPlus, 5, $1-5$.

Kawai, N. \& He, H. (2016). Breaking snake camouflage: Humans detect snakes more accurately than other animals under less discernible visual conditions. PLOS ONE, 11: e0164342.

Kupfer, T. R., \& Le, A. T. (2018). Disgusting clusters: trypophobia as an overgeneralised disease avoidance response. Cognition and Emotion, 32, $729-741$.

Le, A. T., Cole, G. G., \& Wilkins, A. J. (2015). Assessment of trypo- phobia and an analysis of its visual precipitation. Quarterly Journal of Experimental Psychology, 68, 2304-2322.

LeDoux, J. (1998). The emotional brain: The mysterious underpinnings of emotional life. New York: Simon \& Schuster.

Martínez-Aguayo, J. C., Lanfranco, R. C., Arancibia, M., Sepúlveda, E., \& Madrid, E. (2018). Trypophobia: What Do We Know So Far? A Case Report and Comprehensive Review of the Literature. Frontiers in Psychiatry, 9:15.

Murphy, S. T., \& Zajonc, R. B. (1993). Affect, cognition, and awareness: Affective priming with optimal and suboptimal stimulus exposures. Journal of Personality and Social Psychology, 64, 723-739.

Nomura, K., \& Yotsumoto, Y. (2018). Failure to Replicate the Increased Temporal 
Resolution Induced by Images That Give Impression of Danger. Japanese Psychological Research, 60, 179-187.

Rotteveel, M., de Groot, P., Geutskens, A., \& Phaf, R. H. (2001). Stronger suboptimal than optimal affective priming? Emotion, 1, 348-364.

O’Hare, L., \& Hibbard, P. B. (2011). Spatial frequency and visual discomfort. Vision Research, 51, 1767-1777.

Öhman, A., \& Soares, J. J. (1994). "Unconscious anxiety": Phobic responses to masked stimuli. Journal of Abnormal Psychology, 103, 231-240.

Park, J. H., Schaller, M., \& Crandall, C. S. (2007). Pathogen-avoidance mechanisms and the stigmatization of obese people. Evolution and Human Behavior, 28, $410-414$.

Sasaki, K.*, Yamada, Y.*, Kuroki, D., \& Miura, K. (2017). Trypophobic discomfort is spatial-frequency dependent. Advances in Cognitive Psychology, 13, 224-231 (*equal contribution).

Schaller, M. (2011). The behavioural immune system and the psychology of human sociality. Philosophical Transactions of the Royal Society B: Biological Sciences, 366, 3418-3426.

Schaller, M., \& Park, J. H. (2011). The behavioral immune system (and why it matters). Current Directions in Psychological Science, 20, 99-103.

Shirai, R., Banno, H., \& Ogawa, H. (in press). Trypophobic images induce oculomotor capture and inhibition. Attention, Perception, \& Psychophysics.

Stein, T., Seymour, K., Hebart, M. N., \& Sterzer, P. (2014). Rapid fear detection relies on high spatial frequencies. Psychological Science, 25, 566-574.

Sweeny, T. D., Grabowecky, M., Suzuki, S., \& Paller, K. A. (2009). Long-lasting 
effects of subliminal affective priming from facial expressions. Consciousness and Cognition, 18, 929-938.

Tamietto, M., \& de Gelder, B. (2010). Neural bases of the non-conscious perception of emotional signals. Nature Reviews Neuroscience, 11, 697-709.

Tsuchiya, N., Moradi, F., \& Felsen, C. (2009). Intact rapid detection of fearful faces in the absence of the amygdala, Nature Neuroscience, 12, 1224-1225.

Vlok-Barnard, M., \& Stein, D. J. (2017). Trypophobia: An investigation of clinical features. Revista Brasileira de Psiquiatria, 13:0.

Vuilleumier, P. (2005). How brains beware: Neural mechanisms of emotional attention. Trends in Cognitive Sciences, 9, 585-594.

Vuilleumier, P., Armony, J. L., Driver, J., \& Dolan, R. J. (2003). Distinct spatial frequency sensitivities for processing faces and emotional expressions. Nature Neuroscience, 6, 624-631.

Winston, J. S., Vuilleumier, P., \& Dolan, R. J. (2003). Effects of low- spatial frequency components of fearful faces on fusiform cortex activity. Current Biology, 13, 1824-1829.

Yamada, Y., \& Sasaki, K. (2017). Involuntary protection against dermatosis: A preliminary observation on trypophobia. BMC Research Notes, 10:658.

Yang, E., Zald, D. H., \& Blake, R. (2007). Fearful expressions gain preferential access to awareness during continuous flash suppression. Emotion, 7, 882-886. 


\section{Acknowledgments}

The present study was supported by JSPS KAKENHI Grant Number JP17J05236 given to K.S., and JP17H06344 to K.W., and JP15H05709, JP16H03079, JP16H01866, JP17H00875, JP18H04199, and JP18K12015 given to Y.Y., and Core Research for Evolutional Science and Technology Grant Number JPMJCR14E4 to K.W. 


\section{Author contribution}

All of the authors designed the experiment, discussed the data, and wrote the paper.

K.S. conducted the experiment and analyzed the data. 


\section{Competing financial interests}

The authors declare no competing financial interests. 
Figure legends

Figure 1. Schematic representation of a trial in Experiment 1.

Figure 2. Results of Experiment 1. The dotted line indicates the chance level. The error bars denote standard errors of the mean.

Figure 3. Results of Experiment 2. The dotted line indicates the chance level. The error bars denote standard errors of the mean.

Figure 4. Schematic representation of a trial in Experiment 3.

Figure 5. Results of Experiment 3. The $d^{\prime}$ (a) and $c$ (b) of each condition. The error bars denote standard errors of the mean. 


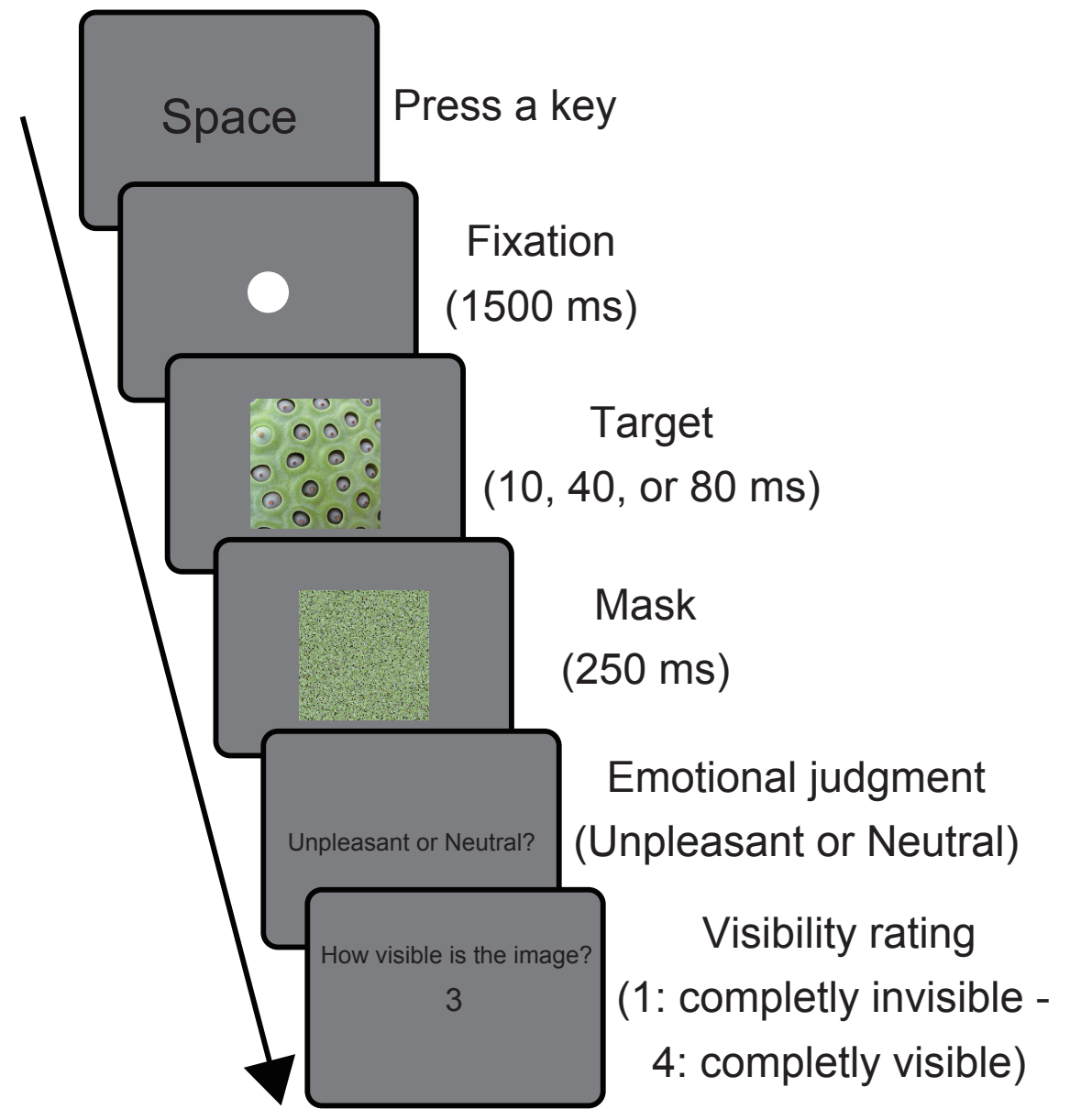

Figure 1 


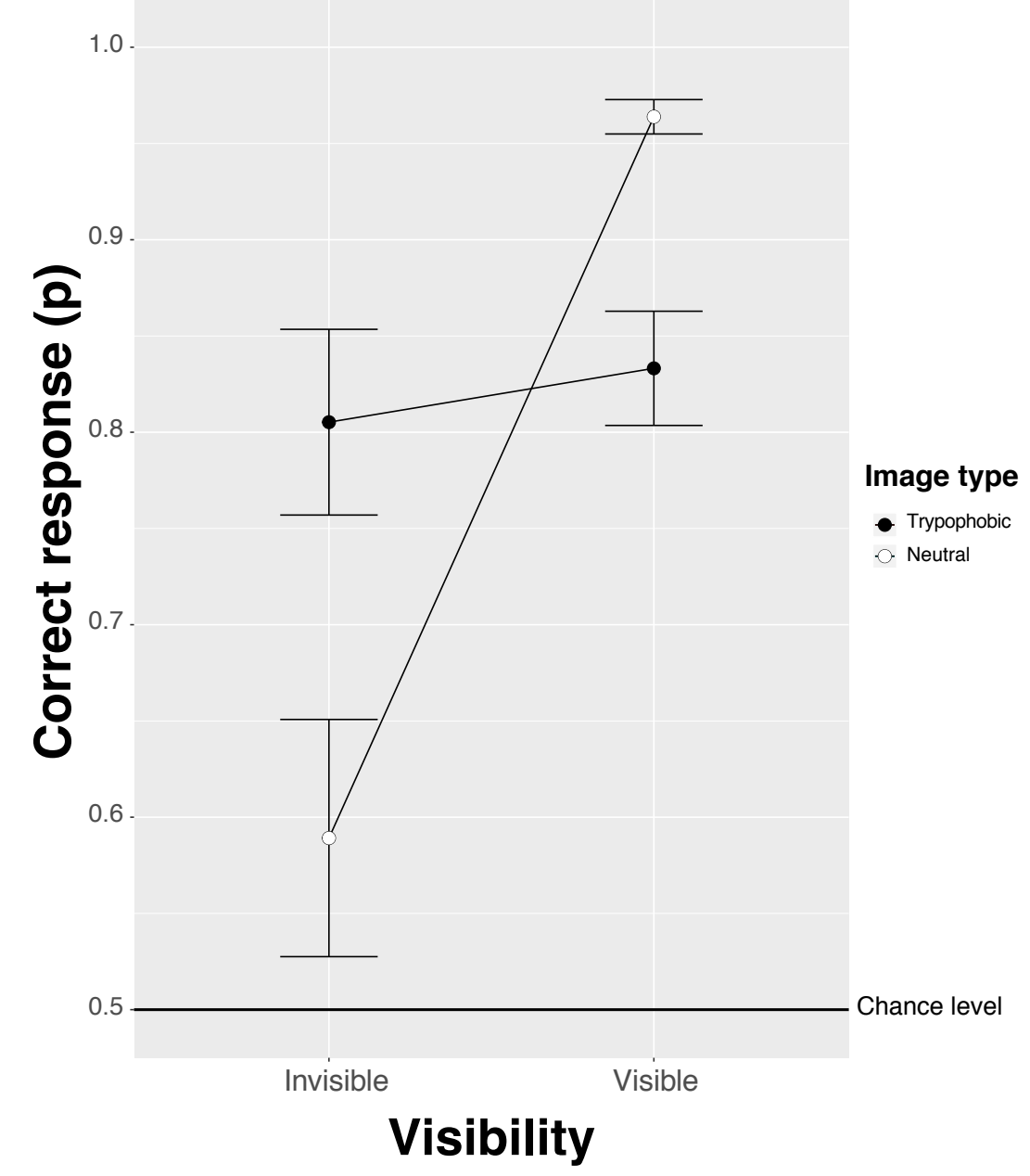

Figure 2 


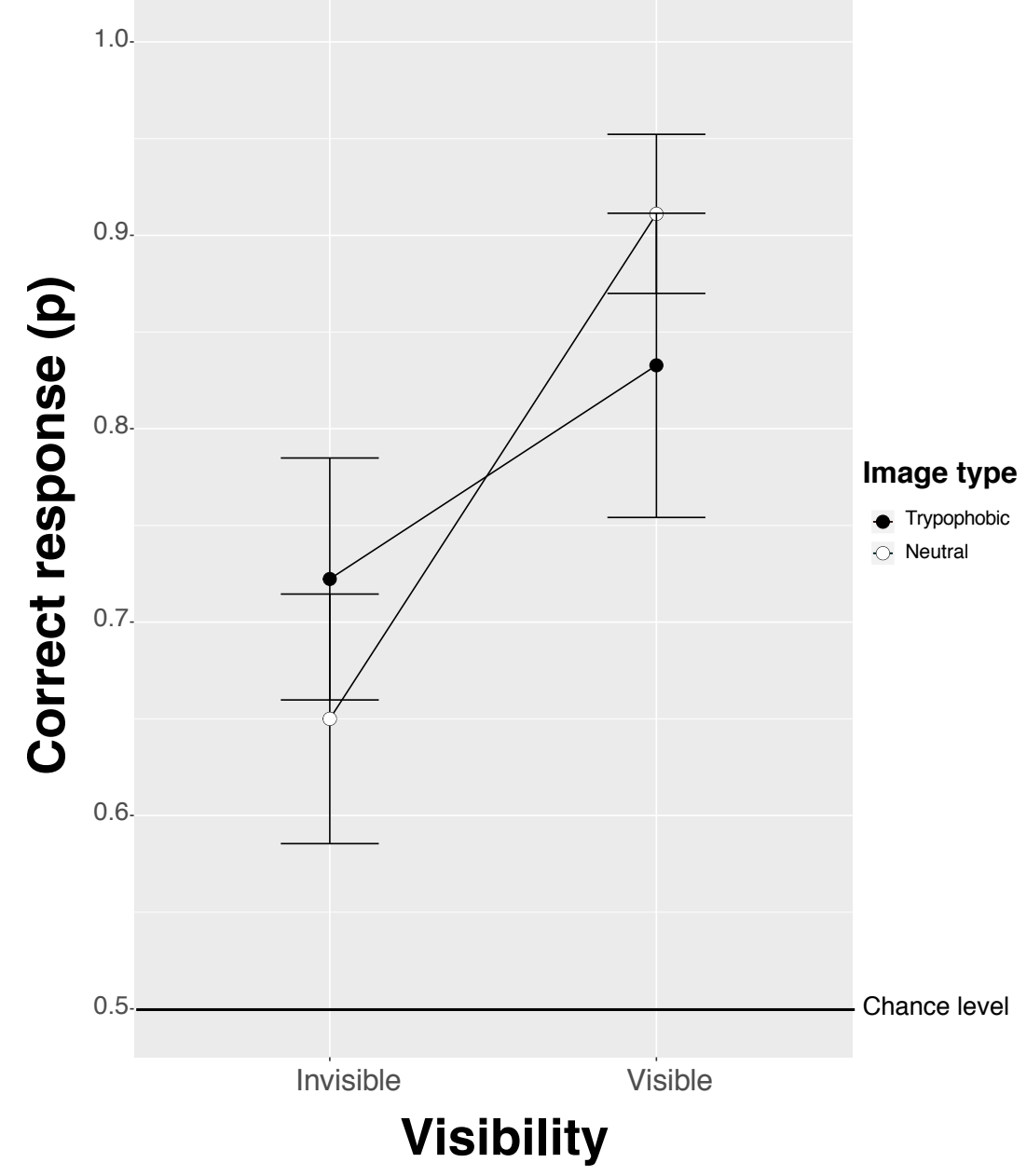

Figure 3 


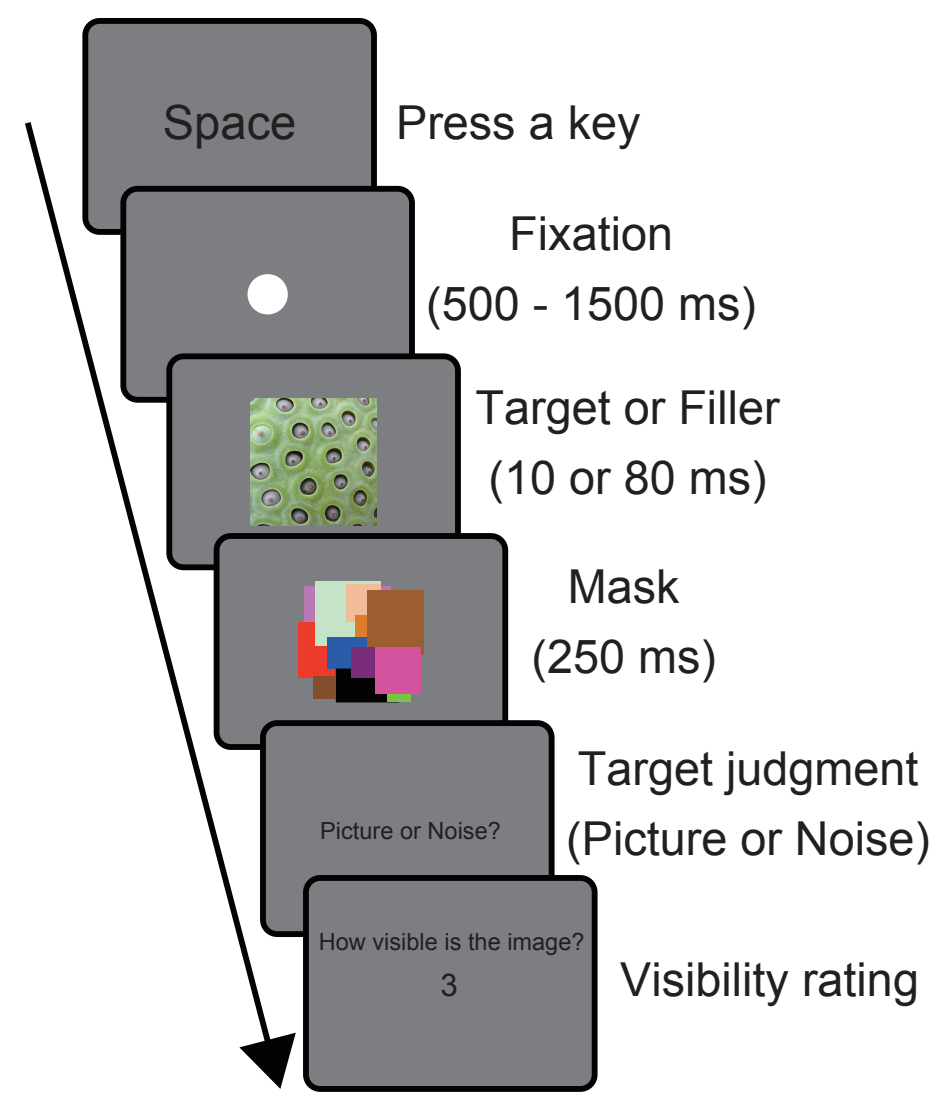

Figure 4 
(a)

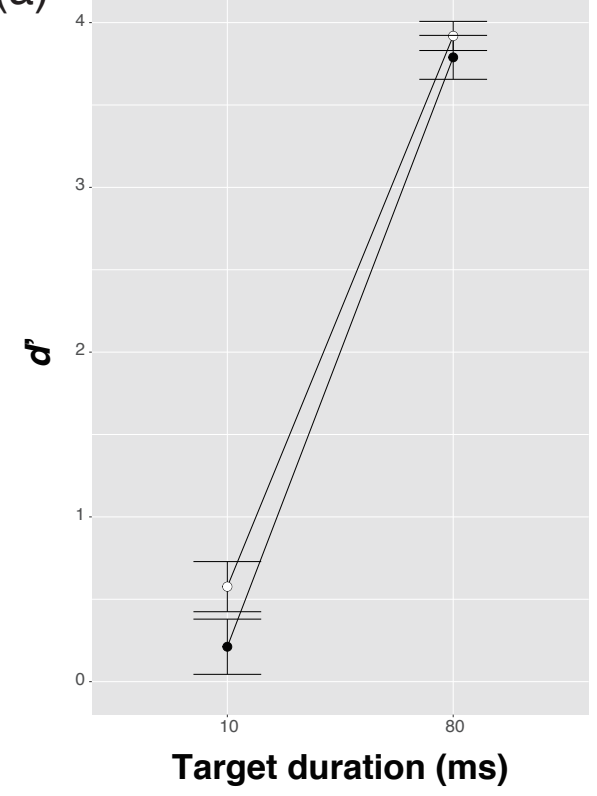

(b)

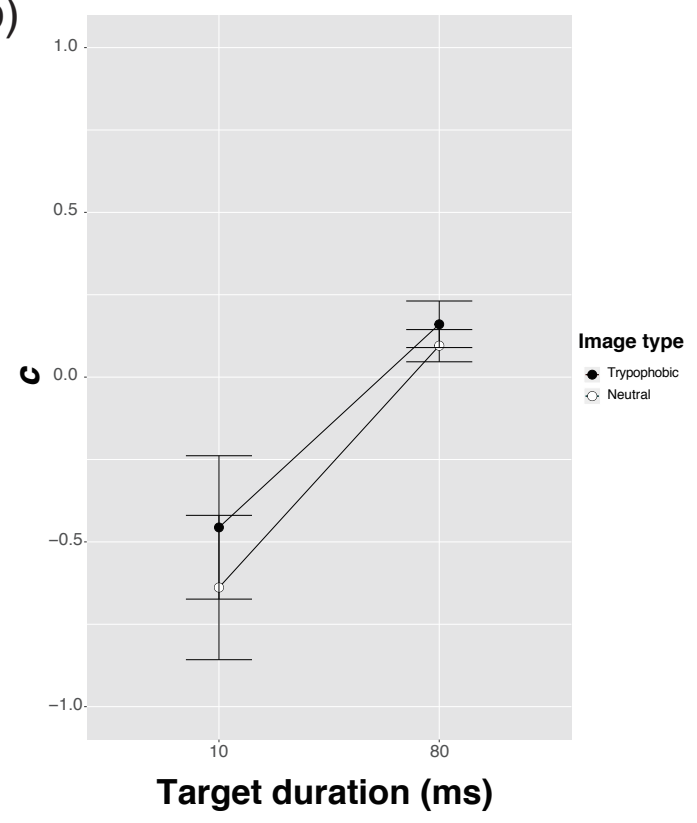

Figure 5 